\title{
Combining an in silico approach with an animal experiment to investigate the protective effect of troxerutin for treating acute lung injury
}

\author{
Ying $\mathrm{Li}^{\mathrm{i}^{*}}$, Pan $\mathrm{Ma}^{1}$, Jin $\mathrm{Fu}^{1}$, Jingjing $\mathrm{Wu}^{2}$ and $\mathrm{Xue} \mathrm{Wu}^{2}$
}

\begin{abstract}
Background: Troxerutin (TRX), a naturally occurring flavonoid in various fruits, has been reported to exhibit numerous pharmacological and biological activities in vitro and in vivo. However, the molecular mechanisms underlying TRX as a treatment for disease are poorly understood.

Methods: Using pharmacophore mapping and inverse docking, a set of potential TRX target proteins that have been associated with multiple forms of diseases was obtained. Bioinformatic analyses were performed using the Enrichr and STRING servers to analyse the related biological processes and protein-protein networks. Furthermore, we investigated the potential protective effect of TRX against lipopolysaccharide-induced acute lung injury (ALI) using a mouse model. Morphological changes in the lungs were assessed using haematoxylin and eosin staining. Inflammatory cytokines, tumour necrosis factor-a (TNF-a), interleukin-1 $\beta$ (IL-1 $\beta$ ), IL-6 and IL-10 were investigated using ELISA. Activation of MAPK and NF-KB was detected using western blotting.

Results: Our network pharmacology analysis revealed the existence of multiple TRX-related chemical-target interactions and the related biological processes. We found that pretreatment with TRX protected against histological changes and obviously regulated the inflammatory cell counts and inflammatory cytokine levels in bronchoalveolar lavage fluid. Based on bioinformatic and western blot analyses, TRX may exert a protective effect against ALI by inhibiting MAPK and NF-KB signalling.

Conclusions: TRX can ameliorate pulmonary injury by inhibiting the MAPK and NF-KB signalling pathways and has a potential protective effect against ALI. This study may be helpful for understanding the mechanisms underlying TRX action and for discovering new drugs from plants for the treatment of ALI.
\end{abstract}

Keywords: Troxerutin, In silico prediction, Acute lung injury, Protective effect, Inflammatory response

\section{Background}

Troxerutin (TRX) is a natural derivative of flavonoid rutin, which is found in grains, fruits and vegetables. Previous studies have shown that TRX has multiple biological functions, such as antioxidative, anti-inflammatory and antiplatelet activities [1-3]. Pretreatment with TRX can induce cardioprotection by modulating the inflammatory response after myocardial ischaemia/reperfusion injury in rat models

\footnotetext{
* Correspondence: liyingjzykd@163.com

1 Laboratory Teaching Center of Basic Medicine, Jinzhou Medical University,

Jinzhou 121001, Liaoning, People's Republic of China

Full list of author information is available at the end of the article
}

[4]. Pre-treatment with TRX can induce cardioprotection through modulating the inflammatory response after myocardial ischaemia/reperfusion injury in rat models [1]. TRX reportedly protects against liver inflammation by attenuating oxidative stress-mediated $\mathrm{NAD}^{+}$depletion [5]. However, whether TRX preconditioning exerts a protective effect on lung injury remains unclear.

Recently, computational approaches in combination with experiments have been successfully applied to natural product research, including the objective elucidation of the mechanisms of action and the comprehensive prediction of effective therapeutic combinations [6-8]. Yang

(c) The Author(s). 2019 Open Access This article is distributed under the terms of the Creative Commons Attribution 4.0 International License (http://creativecommons.org/licenses/by/4.0/), which permits unrestricted use, distribution, and reproduction in any medium, provided you give appropriate credit to the original author(s) and the source, provide a link to the Creative Commons license, and indicate if changes were made. The Creative Commons Public Domain Dedication waiver (http://creativecommons.org/publicdomain/zero/1.0/) applies to the data made available in this article, unless otherwise stated. 
et al. systematically investigated Ginkgo biloba leaves for the treatment of cardio-cerebrovascular diseases in an animal model by incorporating pharmacokinetic pre-screening and network analysis [9]. Twelve active compounds and mechanisms of the Xipayi KuiJie'an enema for the treatment of ulcerative colitis were identified using a systems pharmacology approach [10]. An increasing number of studies have shown that the application of systems pharmacology provides guidance for exploring the therapeutic mechanism of compound and herbal medicine [11-13].

Acute lung injury (ALI) is a common and major cause of acute respiratory failure, characterized by severe inflammation of lung parenchyma [14-17]. Although some new therapeutic strategies have been developed, ALI remains a major cause of mortality $[16,18]$. The major characteristics of ALI are respiratory dysfunction with destruction of the alveolar capillary membrane, subsequent infiltration of peripheral inflammatory cells, and release of several cytokines [19]. Intranasal administration of lipopolysaccharide (LPS) has been widely used to induce pulmonary inflammation in animal models of ALI [20-22]. The exposure of LPS to lung tissue directly induces an acute inflammatory response in the airspaces and lung parenchyma, characterized by oedema and increasing amounts of inflammatory cells and inflammatory cytokines, such as TNF- $\alpha$, IL- $1 \beta$ and IL- 6 , in the bronchoalveolar lavage fluid (BALF).

A systems pharmacology approach was used to investigate the pharmacological mechanisms of TRX in this study. First, the potential molecular targets of TRX were predicted by the PharmMapper and idTarget servers. Second, multiple targets of TRX were analysed using various bioinformatic platforms, such as STRING and DAVID. Finally, the protective effect and underlying mechanisms of TRX on ALI were explored in a mouse model. Our results provide important insights into the efficiency of TRX for treating ALI.

\section{Methods}

\section{Reagents}

TRX (purity > 98\%) was purchased from Nanjing DASF Bio-Technology Co., Ltd. (Nanjing, China). Lipopolysaccharide (LPS, Escherichia coli O111: B4) was provided by Sigma-Aldrich Chemical Company (St. Louis, MO, USA). The dexamethasone (Dex) sodium phosphate injection was purchased from Changle Pharmaceutical Co. (Xinxiang, Henan, China). Interleukin (IL)-1 $\beta$, IL-6, IL-10, and TNF- $\alpha$ levels were quantified by ELISA (Biolegend, San Diego, CA, USA). The ERK, p-ERK, JNK, p-JNK, P38, p-P38 and $\beta$-actin primary antibodies were purchased from $\mathrm{AB}$ Clonal Biotech Co., Ltd. (College Park, MD, USA). The NF-kB p-p65 and p65 primary antibody was purchased from Cell Signaling Technology
Inc. (MA, USA). Horseradish peroxidase (HRP)-labelled anti-mouse and anti-rabbit IgG $(\mathrm{H}+\mathrm{L})$ secondary antibodies were obtained from Beijing Solarbio Science \& Technology Co., Ltd. (Beijing, China).

\section{Prediction of putative targets}

The protein targets of TRX were predicted using the PharmMapper and idTarget servers [23, 24]. The optimized structure of TRX in mol2 format was submitted to PharmMapper and idTarget for prediction of proteins with three dimensional structures in the Protein databank. The lists of predicted targets that were ranked in the top $1-5 \%$ by the abovementioned servers were further annotated to screen out the putative target list pertaining to anti-inflammation activity.

\section{Analysis using the database for annotation, visualization and integrated discovery}

Following the computational prediction of TRX, Enrichr and ClueGO APP in Cytoscape were employed to provide a biological functional interpretation of its predicted potential targets [25, 26]. To elucidate the relevant functions linked to the predicted gene list, the target genes were evaluated by Gene Ontology (GO) using Enrich. For each GO term, the $p$-value of function clustering and the p-value following multiple detection corrections, such as the Benjamini and false discovery rate (FDR) corrections, were calculated. Enrichment scores and Fisher's exact test $P$-values were then calculated to identify which functional gene groups were significantly enriched.

\section{Protein-protein interaction networks}

The protein IDs of these putative protein targets predicted using the PharmMapper and idTarget servers were individually submitted to Search Tool for the Retrieval of Interacting Genes/Proteins v10 (STRING 10) to obtain biological functional interpretations of their targets in Homo sapiens [27]. Pathway enrichment analysis was performed for the Kyoto Encyclopedia of Genes and Genomes (KEGG) enrichment by STRING, and the significantly enriched pathways were identified based on a value of $P<0.05$.

\section{Animals}

Male BALB/C mice were purchased and inbred in a specific pathogen-free (SPF) facility at Jinzhou Medical University. Mice (18-22 g, 8 weeks old) were housed at room temperature $\left(24 \pm 1^{\circ} \mathrm{C}\right)$ in a 12 light/dark cycle. All experimental procedures were performed in accordance with the National Institutes of Health Guide for the Care and Use of Laboratory Animals. The protocols were also approved by the Animal Care \& Welfare Committee of Jinzhou Medical University (approval ID: 
SYXK-liaoning-2017-0003). No mice died, and no apparent signs of exhaustion were observed during the experimental period.

\section{Experimental protocols}

After adjusting to the environment, the mice were randomly divided into the following 4 groups, with 18 mice in each group: control, LPS, TRX $(150 \mathrm{mg} / \mathrm{kg})+\mathrm{LPS}$, and Dex $(5 \mathrm{mg} / \mathrm{kg})+$ LPS. In the group receiving TXR, the mice were treated with $150 \mathrm{mg} / \mathrm{kg}$ TRX in distilled water containing $0.1 \%$ Tween 80 via oral gavage once per day for 7 days consecutively. In the control and LPS groups, the mice were treated with sterile distilled water containing the TRX solvent via oral gavage daily for one consecutive week. One hour before LPS instillation, Dex (5 mg/kg) dissolved in PBS was administered intraperitoneally as a positive control. Mice from the LPS, TRX + LPS and Dex + LPS groups were anaesthetised with pentobarbital sodium $(45 \mathrm{mg} / \mathrm{kg}$, intraperitoneal injection) and intranasally administered $25 \mu \mathrm{g}$ of LPS, which was diluted in PBS. Control mice were given PBS without LPS. Twenty-four hours after PBS or LPS treatment, the animals were euthanized by $\mathrm{CO}_{2}$ gas. The TRX and Dex dosages used in this study were based on previously published literature [28-33].

\section{Bronchoalveolar lavage fluid analysis}

Bronchoalveolar lavage (BAL) was performed by three consecutive instillations of $0.5 \mathrm{ml}$ of PBS $(n=6-8$ in each group). The BAL fluid samples were centrifuged at $800 \times \mathrm{g}$ for $10 \mathrm{~min}$ at $4{ }^{\circ} \mathrm{C}$ and collected for further analysis. The cell pellets were resuspended in PBS and stained using a Wright-Giemsa staining kit (Solarbio Life Sciences, Inc.). The concentrations of TNF- $\alpha$, IL-1 $\beta$, IL- 6 and IL-10 in the supernatants of the BALF were measured using commercially available ELISA kits in accordance with the manufacturer's protocol (Biolegend, San Diego, CA, USA). Total protein concentrations in the BALF were quantified using the BCA Protein Assay Kit.

\section{Histological examination of lung tissues}

The mouse lung tissues ( $n=5$ per group) were fixed overnight in $10 \%$ buffered formalin, embedded in paraffin, sectioned at a thickness of $8 \mu \mathrm{m}$, and then stained with haematoxylin and eosin (H\&E). Pathological changes in lung tissues were observed under a Nikon microscope (Nikon, Japan) using ImageJ software (NIH, Bethesda, MD, USA).

\section{Pulmonary oedema level}

The lung tissues were separated from the mice, and their weights were recorded. Subsequently, the lung tissues were subsequently incubated at $90^{\circ} \mathrm{C}$ for $24 \mathrm{~h}$ and weighed to obtain the dry weight $(n=5-7$ in each group). Then, the lung W/D ratio was calculated $[32,33]$.

\section{Western blot analysis}

Lung samples were lysed with lysis buffer (Beyotime, Shanghai, China). Total proteins were purified by sodium dodecyl sulfate polyacrylamide gel electrophoresis and transferred to a polyvinylidene difluoride membrane. After blocking with $5 \%$ nonfat milk, proteins were incubated with specific primary antibodies at $4{ }^{\circ} \mathrm{C}$ overnight. After washing three times with TBST, the membrane was incubated for an additional $2 \mathrm{~h}$ with an HRP-conjugated secondary antibody at room temperature. Finally, proteins were detected by enhanced chemiluminescence (ECL, GE Healthcare, Buckinghamshire, UK).

\section{Statistical analysis}

Data are presented as the mean \pm SEM from three independent experiments. Statistics were calculated using GraphPad Prism 5 software (GraphPad Software, San Diego, CA, USA). One-way analysis of variance (ANOVA) was utilized to compare data among multiple groups $(P<0.05$ set as a standard to indicate significance).

\section{Results}

\section{Potential targets of TRX}

Potential targets of TRX identified by dual virtual screening procedures were analysed. Reverse pharmacology mapping was conducted using the PharmMapper server to identify potential targets of TRX with the submitted molecular structure. A list of the top 100 potential targets was obtained and further annotated to screen out the targets associated with diseases (Additional file 1: Table S1). Among the top 100 targets predicted by PharmMapper, 34 proteins belong to the species Homo sapiens, and among the top 500 targets predicted by idTarget, 135 proteins belong to the species Homo sapiens (Additional file 1: Table S1); these results are shown in Fig. 1b.

\section{GO analysis of predicted TRX targets}

GO analysis was performed to classify the predicted targets of TRX and to identify the most significantly enriched GO terms. GO enrichment can directly reflect the distribution of target genes for each enriched $\mathrm{GO}$ term in the categories of biological process (BP), cellular component (CC) and molecular function (MF). As shown in Fig. 2a, some BPs were enriched, such as negative regulation of protein kinase activity by protein phosphorylation (GO:0100002), cellular response to vitamin $\mathrm{E}$ (GO:0071306), positive regulation of tubulin deacetylation (GO:0090044), regulation of dipeptide transmembrane transport (GO:2001148), positive regulation of tubulin 


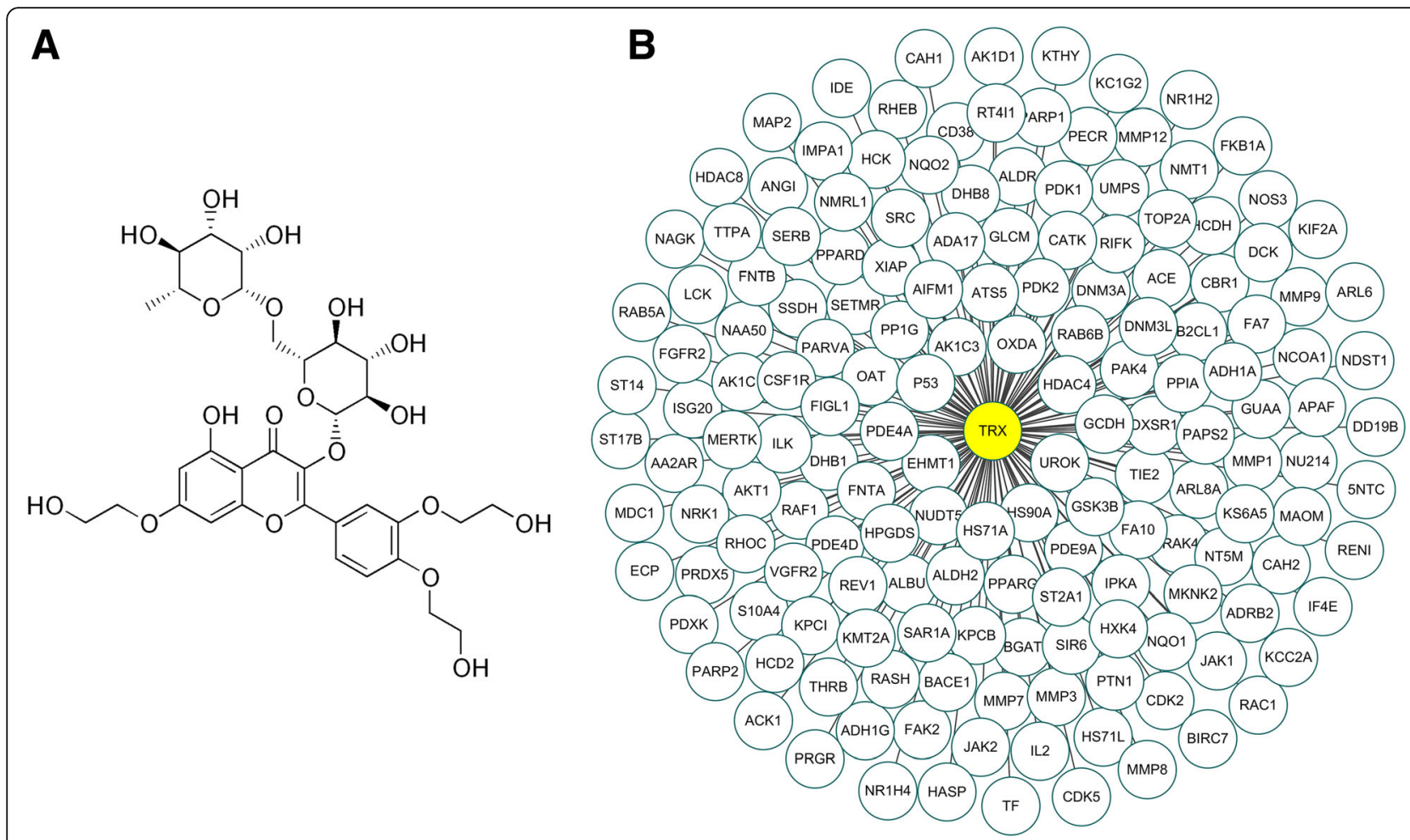

Fig. 1 The schematic structure and the predicted target proteins of troxerutin (TRX). (a) The chemical structure of TRX. (b)162 potential targets of TRX that belong to species Homo sapiens had binding energies ranked in the top 100 from PharmMapper and the top 500 from idTarget

deacetylation (GO:0090044), and negative regulation of relaxation of cardiac muscle (GO:1901898).

In addition, the top ten MF terms, shown in Fig. 2b, were as follows: protein kinase activity (GO:0004672), kinase activity (GO:0016301), protein kinase binding (GO:0019901), protein tyrosine kinase activity (GO:0004713), phosphotransferase activity, alcohol group as acceptor (GO:0016773) and nonmembrane spanning protein tyrosine kinase activity (GO:0004715). The main subcategories of the CC group were cytoplasmic side of membrane (GO:0098562), ruffle membrane (GO:0032587), focal adhesion (GO:0005925) and basal plasma membrane (GO:0009925).

\section{Protein-protein networks and pathway analysis of the predicted TRX targets}

To identify functional connections between the predicted proteins, protein-protein interactions were predicted using STRING-db. The human proteins and the functional interactions between them are shown in Fig. 3a. The coloured lines between the proteins indicate various types of evidence for interactions.

Next, pathway enrichment analysis was conducted. The STRING server identified 74. KEGG pathways that were significantly enriched in the target list derived from PharmMapper and idTarget (Addtional file 2: Table S2). The signalling pathways that participate in airway inflammation, including the MAPK signalling pathway
(KEGG ID: 04010), VEGF signalling pathway (KEGG ID: 04370), and $\mathrm{T}$ cell receptor signalling pathway (KEGG ID: 04660), were found in the KEGG enrichment analysis of putative targets. In particular, MAPK signalling was ranked in the top nine signalling pathways (Fig. 3b), and nine predicted targets in the MAPK pathway may interact with TRX; these targets are labelled as red nodes in the network (Fig. 3a).

Following the bioinformatic prediction of the molecular TRX interactome, we examined the effect of TRX on ALI by assessing the inflammatory response in vivo.

\section{TRX modulates the pathophysiological features of LPS-induced ALI}

As shown in Fig. 4, characteristic morphological changes, including alveolar wall thickening and obvious inflammatory cell infiltration, were observed in lung sections after LPS administration. These changes in the histopathological features of ALI were much less pronounced in the TRX pretreatment $(150 \mathrm{mg} / \mathrm{kg})$ and Dex $(5 \mathrm{mg} / \mathrm{kg})$ groups. Our results suggest that TRX and Dex significantly attenuated lung damage in LPS-induced ALI mice.

TRX ameliorates the LPS-induced increases in lung oedema and microvascular protein permeability

As shown in Fig. 5a, the lung W/D ratio was higher after LPS stimulation than after the control treatment. 


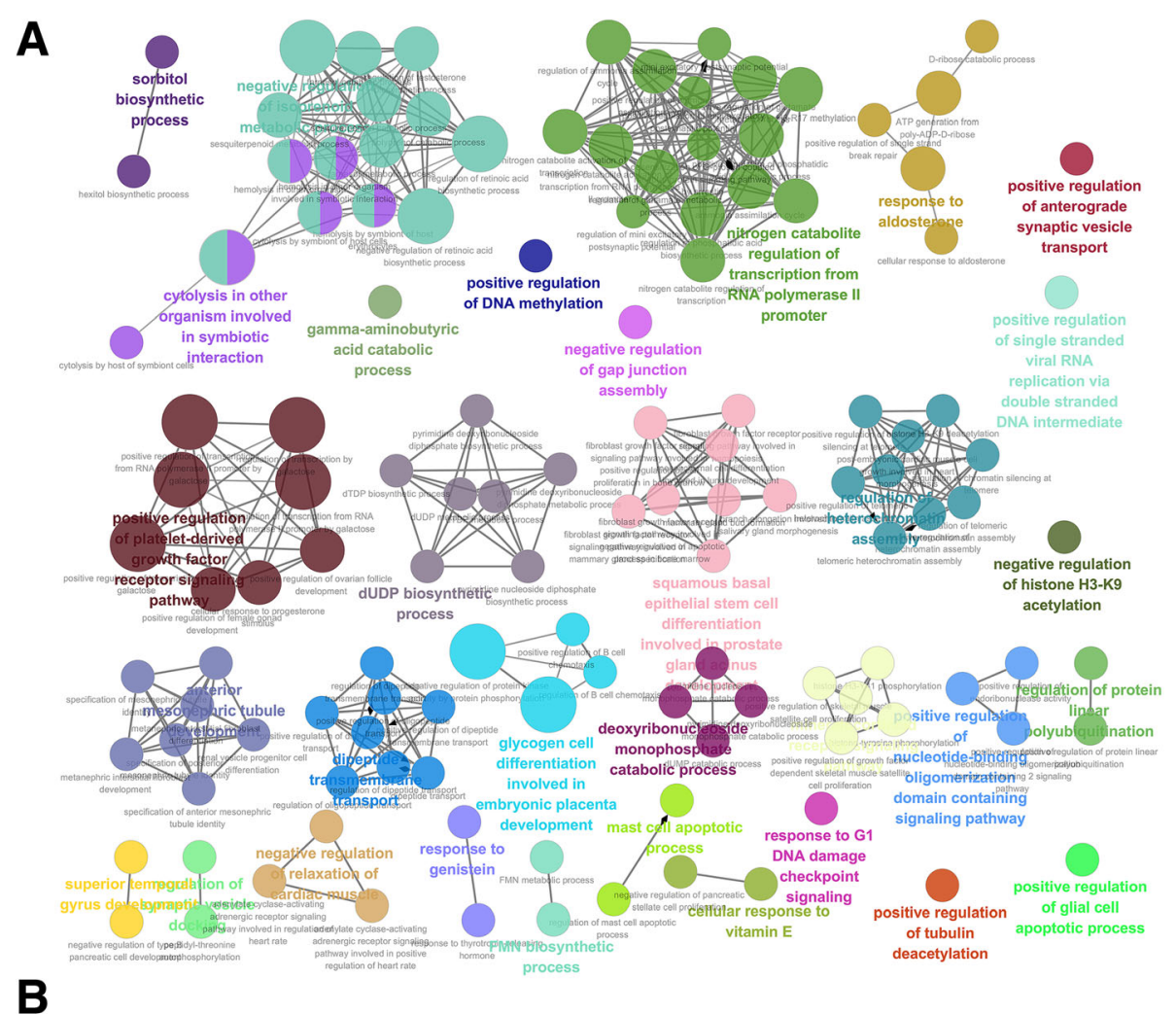

B

\section{protein kinase activity (GO:0004672)}

\section{kinase activity (GO:0016301)}

protein kinase binding (GO:0019901)
protein tyrosine kinase activity (GO:0004713)

phosphotransferase activity, alcohol group as acceptor (GO:0016773)

non-membrane spanhing protein tyrosine kinase activity (GO:0004715)

protein homodimerization activity (GO:0042803)

oxidoreductase activity, acting on $\mathrm{NAD}(\mathrm{P}) \mathrm{H}$, quinone or similar compound as acceptor (GO:0016655)

transition metal ion binding (GO:0046914)

mitogen-activated protein kinase kinase binding (GO:0031434)

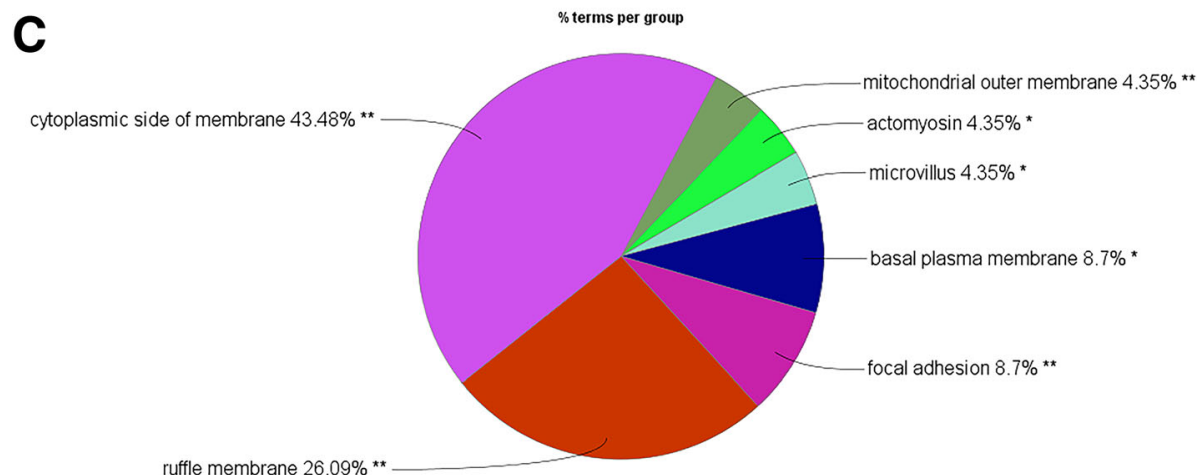

Fig. 2 The GO analysis of predicted targets of TRX. Enrichr analysis was performed to identify the most significantly enriched GO terms. The enriched biological process terms (a), molecular function terms (b) and cellular component (c) were listed based on the $P$-Value 


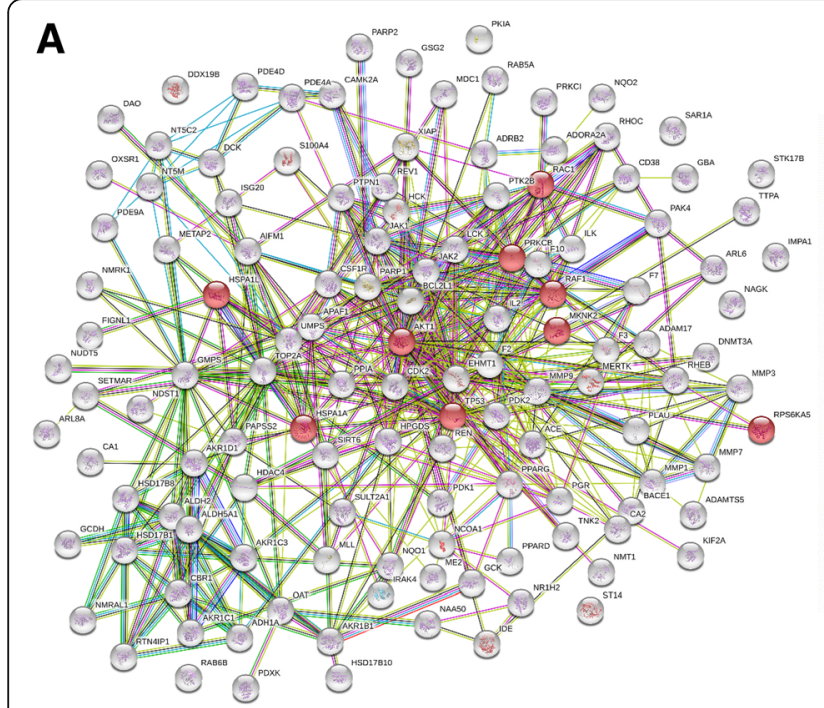

B

Fig. 3 Network pharmacology analysis through the protein interaction of predicted protein targets (a) and signaling pathways (b) of representative ingredients of TRX. The network nodes were predicted proteins and the edges represented the functional associations. The red nodes were proteins included in the MAPK signaling. Pathways with a $p$ value $\leq 0.05$ after FDR correction were considered significant

Pretreatment with TRX $(150 \mathrm{mg} / \mathrm{kg})$ and Dex $(5 \mathrm{mg} / \mathrm{kg})$ suppressed the increase in the lung W/D ratio induced by LPS. In addition, LPS administration increased the total protein concentration in BALF compared with that achieved with control treatment. In contrast, administration of TRX or Dex prior to LPS treatment reduced the increase in the total protein concentration (Fig. 5b). These results suggest that TRX was effective in preventing lung oedema and suppressing protein leakage from the lungs of LPS-induced ALI mice.
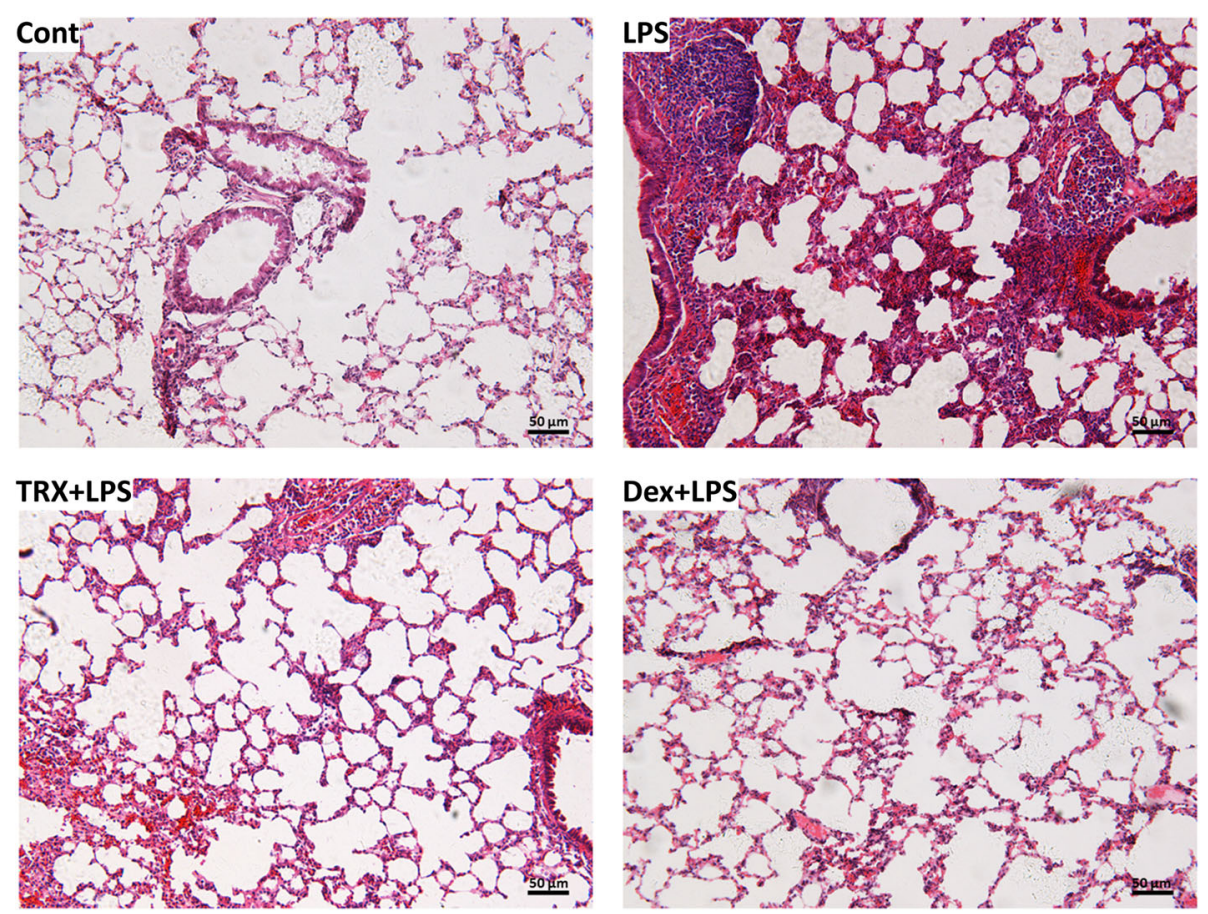

Fig. 4 The effect of TRX on the microscopic appearances of lung from LPS-induced ALI mouse; Cont: a control group, LPS: LPS group, TRX + LPS: LPS + TRX (150 mg/kg) group, Dex + LPS: LPS + Dex $(5 \mathrm{mg} / \mathrm{kg})$ group. Histopathological sections were stained by H.E. Original magnification $\times 200$. $(n=5$ in each group) 

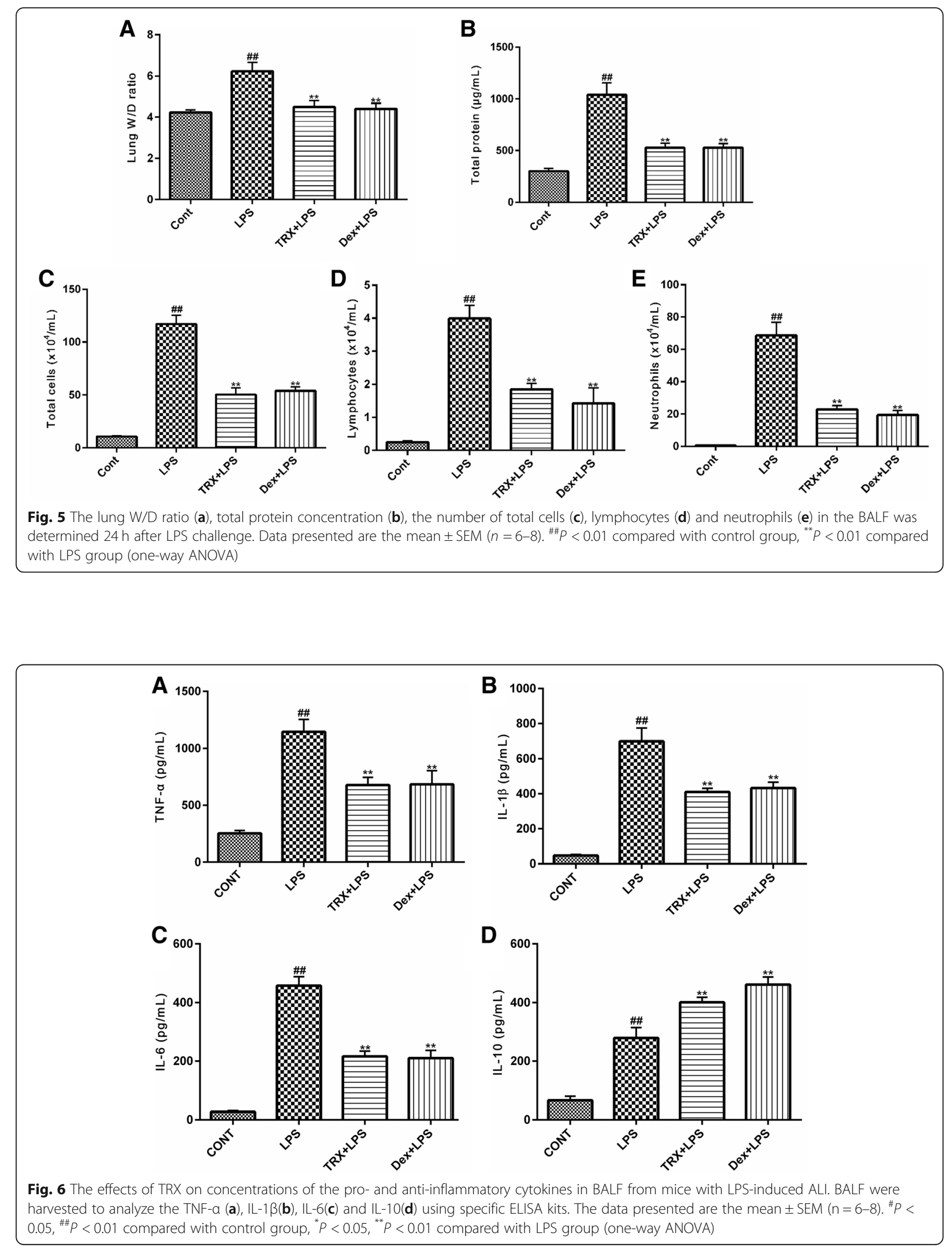


\section{Effects of TRX on lung inflammatory cell accumulation in BALF}

As shown in Fig. 5c-e, the accumulation of total cells, neutrophils and lymphocytes was increased after the LPS challenge compared with that achieved with control treatment. TRX $(150 \mathrm{mg} / \mathrm{kg})$ pretreatment notably reduced the total cell, neutrophil and lymphocyte counts. Our results indicate that TRX effectively suppresses LPS-induced inflammatory cell accumulation.

Effects of TRX on the pro- and anti-inflammatory cytokine and chemokine levels in BALF

To assess the effects of TRX on inflammatory cytokines in LPS-stimulated ALI mice, the levels of IL-1 $\beta$, TNF- $\alpha$, IL- 6 and IL-10 in BALF were measured by ELISA. As shown in Fig. 6 , the levels of IL- $1 \beta$, TNF- $\alpha$, and IL- 6 were significantly increased after LPS inhalation compared with those in the control group. TRX pretreatment dramatically reduced the release of TNF- $\alpha$, IL- $1 \beta$ and IL- 6 in BALF. However, the release of IL-10, an endogenous anti-inflammatory cytokine, was enhanced in the TRX and Dex groups compared with those in the LPS group. Our results suggest that TRX suppressed the release of proinflammatory cytokines and promoted the secretion of anti-inflammatory cytokines.
Effects of TRX on MAPK and NF-KB activation

As shown in Fig. 7a, the total protein levels of ERK, p38 and JNK in lung tissues remained unchanged in the LPS, TRX + LPS, and Dex + LPS groups. LPS stimulation significantly increased the phosphorylation levels of ERK, p38 and JNK. However, pretreatment with TRX or Dex markedly suppressed the phosphorylation of ERK, p38 MAPK and JNK. In addition, the phosphorylation of NF- $\mathrm{kB}$ p65 was markedly increased in the LPS group compared with that in the control group. However, the phosphorylated p65 proteins were reduced in the TRX and Dex groups compared with those in the LPS group (as shown in Fig. 7b). These results suggest that the beneficial effects of TRX may be partly mediated by the MAPK and NF-kB pathways.

\section{Discussion}

In silico methodologies have become a valuable technique in drug discovery, and their impact on drug development is likely to expand [34]. Recently, a novel reverse pharmacology protocol involving a structure-based pharmacophore and inverse docking was proven to be efficient as a rapid and inexpensive method for identifying molecular targets $[6,7]$. The PharmMapper online tool is a web server for

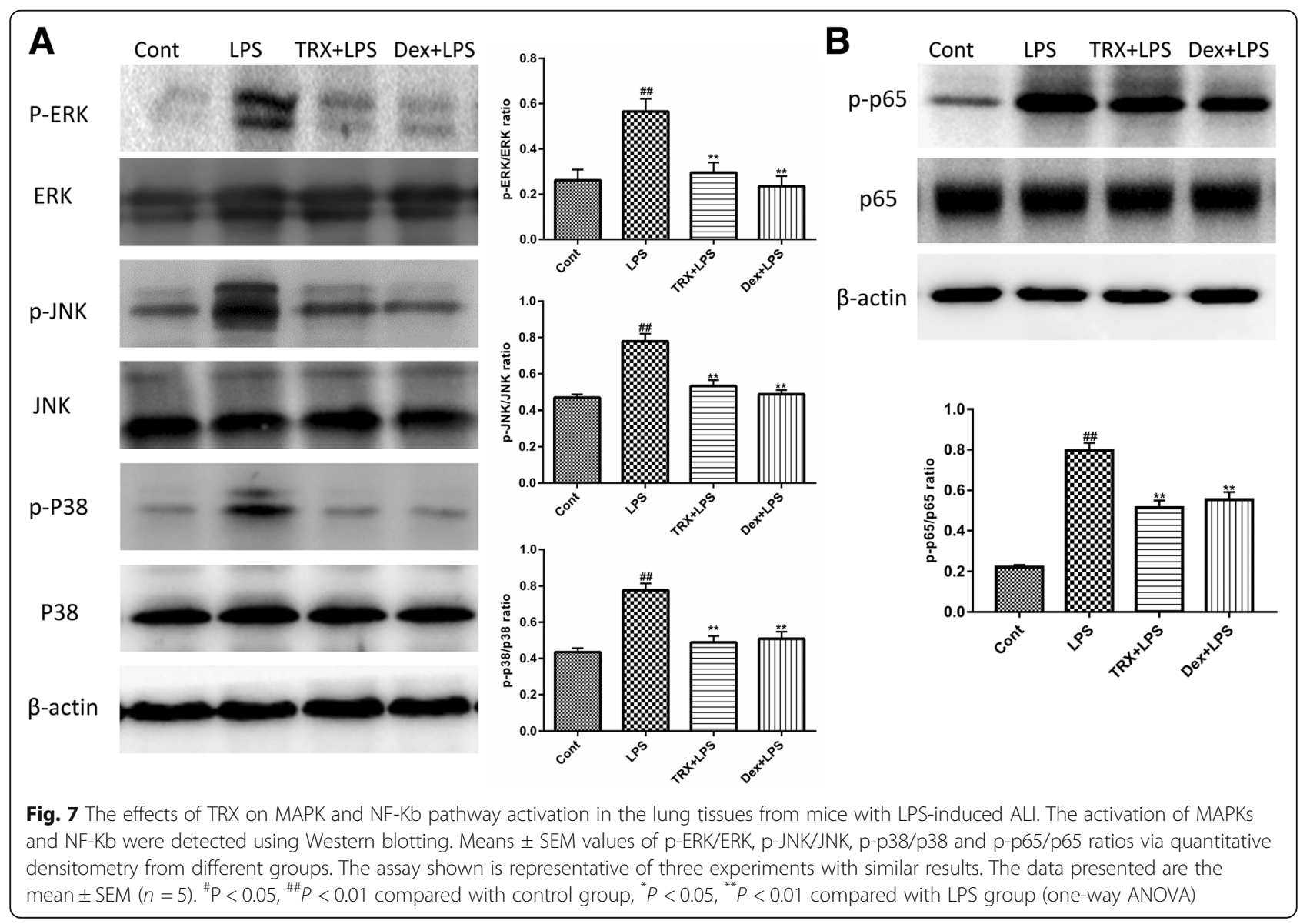


potential drug target identification via reverse pharmacophore matching of the query compound against an in-house pharmacophore model database [23]. The inverse docking approach, in which ligands are docked against the protein targets in databases and the targets are scored according to the docking evaluation, has been successfully explored for target prediction [7, 35-37]. The idTarget server conducts a computer-automated search of potential targets and identifies the drug repositioning potential via inverse docking [24]. In the present study, we obtained and analysed the potential disease-related targets of TRX predicted by PharmMapper and idTarget. Our results show that TRX can modulate several signaling pathways that have important roles in the regulation of various BPs. Consistent with the bioinformatic data obtained in the present study, some previous studies regarding the mechanisms underlying TRX-regulated pharmacological effects have confirmed several TRX targets and TRX-related signalling pathways [3840]. We found that both SRC and Akt were predicted to be possible direct protein targets of TRX according to the PharmMapper and idTarget servers, and the insulin signaling pathway (KEGG ID: 4910) was also enriched in the pathway analysis performed using STRING. Consistent with our results, Sampath reported that TRX may play a significant role in the management of type 2 diabetes mellitus by improving insulin signaling molecules, including the SRC, Akt and GLUT4 proteins [41]. In addition, TRX was reported to have anti-cancer effects, and the pathways in cancer (KEGG ID: 04064) and the NF-кB pathway (KEGG ID: 04064) were predicted to be modulated by TRX in our study [42]. A recent study reported that TRX potentiates 5 -fluorouracil treatment in human gastric cancer by suppressing the STAT3/NF- $\mathrm{KB}$ and $\mathrm{Bcl}-2$ signaling pathways [2]. A recent report indicated that TRX can exert neuroprotective effects in a 6-hydroxydopamine lesion rat model of Parkinson's disease through the PI3K/ER $\beta$ signaling pathway (KEGG ID: 04151) [43], which was also enriched in the computational screen in our study. These data provide preliminary evidence for the validation of predicted molecular targets of TRX using a bioinformatics approach.

Several studies have noted that TRX is a potent anti-inflammatory agent, and TRX has been shown to modulate the in vivo and in vitro inflammatory responses in various disease models [44-46]. The anti-inflammatory effect of TRX has been demonstrated in D-gal-induced kidney injury through inhibition of the $\mathrm{NF}-\kappa \mathrm{B}$ mediated inflammatory response [47]. Another study reported that TRX protects against BDE-47-induced liver inflammation by attenuating oxidative stress-mediated $\mathrm{NAD}^{+}$depletion [5]. In the present study, we investigated the protective effect of TRX against lung injury via bioinformatic analysis and an animal experiment. To this end, we induced ALI in mice via the intranasal administration of LPS, inducing microvascular injury, diffuse alveolar damage and neutrophil infiltration, pathological features similar to those of ALI observed in humans [16]. TRX decreased the lung W/D ratio and total protein concentration, indicating that it could effectively reduce pulmonary oedema in LPS-induced ALI mice. Activated neutrophils and alveolar macrophages induce extensive lung inflammation, resulting in destruction of the basement membrane and altered permeability of the alveolar-capillary membrane $[16,20]$. In the present study, TRX suppressed the recruitment of inflammatory cells in the BALF of ALI mice. In addition, these results were supported by the histological analysis of the lungs, which revealed that the histological injuries were notably attenuated in mice administered TRX, suggesting that TRX might exert a significantly beneficial effect on LPS-induced lung injury.

Considerable evidence has emerged to indicate that restoring the pro- and anti-inflammatory balance is a potential strategy to prevent ALI [48-50]. In BALF from ALI patients, elevated concentrations of TNF- $\alpha$, IL- $1 \beta$, and IL- 6 have been observed and correspond to an unfavourable prognosis [14]. IL-10 is capable of inhibiting the synthesis of proinflammatory cytokines, such as TNF- $\alpha$ and IL- $1 \beta$, and lower levels of IL-10 have been observed in lungs with injury compared to those in healthy lungs [48]. In the present study, we found that TRX may protect against LPS-induced ALI by reducing the production of TNF- $\alpha$, IL- $1 \beta$, and IL- 6 and increasing the levels of IL-10. This result is consistent with those of previous studies reporting potent regulation of inflammatory cytokine production by TRX in postconditioned rats [1].

Airway inflammation is a complex process that involves various signalling pathways, such as the MAPK, NF-kB and VEGF pathways $[19,49,51]$. In our present study, the MAPK and NF-kB signaling pathways were enriched in the set of predicted proteins. Previous research has demonstrated that MAPKs and NF- $k B$ are important mediators involved in the release of cytokines and are associated with the pathogenesis of inflammatory processes in ALI [51-53]. Additionally, GO analysis identified "protein phosphorylation", which is essential for MAPK activation, as a top five GO BP term. Therefore, based on this evidence, we hypothesized that the MAPK and NF- $\mathrm{kB}$ signalling pathways may be involved in the anti-inflammatory effect of TRX on LPS-induced ALI. In the present study, we demonstrated that the phosphorylation of ERK, JNK and p38 can be induced by LPS and that pretreatment with TRX blocks the activation of MAPKs. Consistently, previous studies reported that TRX can suppress the phosphorylation of JNK in a rat model of type 2 diabetes and block TRAF2-ASK1-JNK signalling in the mouse liver $[5,54]$. $\mathrm{NF}-\mathrm{kB}$, which also serves as a major transcription factor, has been reported to play vital roles in the regulation of 
inflammatory cytokine production. Generally, once stimulated, the activation of NF- $\mathrm{KB}$ leads to the increased release of various proinflammatory cytokines, such as IL-1 $\beta$, IL- 6 and TNF- $\alpha$. Therefore, it is important to inhibit NF- $\mathrm{kB}$ activation in many inflammatory diseases. In our present study, TRX administration suppressed phosphorylated p65 in LPS-stimulated ALI. However, further studies are necessary to test the effects of TRX on other pathways and their interactions.

\section{Conclusions}

In summary, potential receptors of TRX were predicted via the PharmMapper and idTarget servers and analysed with Enrichr and STRING. TRX-related signaling pathways were obtained, and multi-scale pharmacological mechanisms of TRX were interpreted. Then, we experimentally demonstrated that TRX exerts a protective effect against LPS-induced ALI by inhibiting the MAPK and NF- $\mathrm{kB}$ signaling pathways. The present study provides an alternative method for the rapid identification of targets in the study of TRX or other herbal medicines and supports the potential clinical application of TRX in treating ALI.

\section{Additional files}

Additional file 1: Table S1. The potential targets of TRX predicted by PharmMapper and idTarget. The top 100 best-fitted hits in PharmMapper with appropriate target annotations, as well as the aligned poses of the respective molecules are outputted. The interactome of TRX was predicated employing the idTarget server via molecular docking of a small compound across human proteome. The Docking Score fields represent the interaction strength of the molecule to the protein and the top 500 was listed. (XLSX $72 \mathrm{~kb}$ )

Additional file 2: Table S2. The results of KEGG pathway enrichment analysis. To identify functional connections between the proteins identified as predicted proteins, pathway enrichment analysis was conducted by STRING servers. The KEGG pathways were significantly enriched in the targets list derived from PharmMapper and idTarget. (XLSX $16 \mathrm{~kb})$

\section{Abbreviations}

ALI: acute lung injury; BALF: Bronchoalveolar lavage fluid; DAVID: Database for Annotation, Visualization and Integrated Discovery; Dex: dexamethasone; ERK: extracellular regulated protein kinases; GO: Gene Ontology; H\&E: haematoxylin and eosin; IL: interleukin; JNK: c-Jun N-terminal kinase; KEGG: Kyoto Encyclopedia of Genes and Genomes; LPS: lipopolysaccharide; MAPK: mitogen-activated protein kinase; PPI: Protein-protein interaction; TNF: Tumor Necrosis Factor; TRX: Troxerutin

\section{Acknowledgements}

All authors thank the research team members and Jinzhou Medical University in the study.

\section{Funding}

This research did not receive any specific grant from funding agencies in the public, commercial, or not-for-profit sector.

\section{Availability of data and materials}

We have presented all our main data in the form of figures and additional file. The datasets supporting the conclusions of this article are included within the article.

\section{Authors' contributions}

LY conceived and designed the experiments; LY, MP and FJ performed the experiments and wrote the paper; MP, WJJ and WX analyzed the data. All authors have read and approved the final manuscript.

\section{Ethics approval and consent to participate}

In according with the Guide for the Animal Care and Use of Laboratory Animals published by the National Institutes of Health (NIH Publications No. 85-23, revised 1996), all experimental protocols and animal handling procedures were approved by the Animal Care Committee and Use Committee of Jinzhou Medical University.

\section{Consent to publication}

Not applicable.

\section{Competing interests}

The authors declare that they have no competing interests.

\section{Publisher's Note}

Springer Nature remains neutral with regard to jurisdictional claims in published maps and institutional affiliations.

\section{Author details}

${ }^{1}$ Laboratory Teaching Center of Basic Medicine, Jinzhou Medical University, Jinzhou 121001, Liaoning, People's Republic of China. ${ }^{2}$ Dalian Institute of Chemical Physics, Chinese Academy of Sciences, 457 Zhongshan Rd., Dalian, People's Republic of China.

Received: 6 November 2018 Accepted: 26 April 2019

Published online: 10 June 2019

\section{References}

1. Badalzadeh R, Baradaran B, Alihemmati A, Yousefi B, Abbaszadeh A. Troxerutin preconditioning and ischemic Postconditioning modulate inflammatory response after myocardial ischemia/reperfusion injury in rat model. Inflammation. 2017:40:136-43.

2. Xu GY, Tang XJ. Troxerutin (TXN) potentiated 5-fluorouracil (5-Fu) treatment of human gastric cancer through suppressing STAT3/NF-KB and $\mathrm{BCl}-2$ signaling pathways. Biomed Pharmacother. 2017;92:95-107.

3. Diba R, Mohaddes G, Mirzaie BF, Farajdokht F, Bayandor P, Hosseindoost M, Mehri K, Zavvari OZ, Babri S. Protective effects of troxerutin on maternal high-fat diet-induced impairments of spatial memory and apelin in the male offspring. Iran J Basic Med Sci. 2018;21(7):682-7.

4. Basile M, Gidaro S, Pacella M, Biffignandi PM, Gidaro GS. Parenteral troxerutin and carbazochrome combination in the treatment of posthemorrhoidectomy status: a randomized, double-blind, placebo-controlled, phase IV study. Curr Med Res Opin. 2001;17:256-61.

5. Zhang ZF, Shan Q, Zhuang J, Zhang YQ, Wang X, Fan SH, Lu J, Wu DM, Hu $B$, Zheng YL. Troxerutin inhibits 2,2',4,4'-tetrabromodiphenyl ether (BDE-47)induced hepatocyte apoptosis by restoring proteasome function. Toxicol Lett. 2015;233:246-57.

6. Kibble M, Saarinen N, Tang J, Wennerberg K, Makela S, Aittokallio T. Network pharmacology applications to map the unexplored target space and therapeutic potential of natural products. Nat Prod Rep. 2015;32:1249-66.

7. Guney E, Menche J, Vidal M, Barabasi A. Network-based in silico drug efficacy screening. Nat Commun. 2016;7.

8. Wang B, Xiao Y, Yang X, He Y, Jing T, Wang W, Zhang J, Lin R. Protective effect of dihydromyricetin on LPS-induced acute lung injury. J Leukoc Biol. 2018.

9. Yang Y, Li Y, Wang J, Sun K, Tao W, Wang Z, Xiao W, Pan Y, Zhang S, Wang Y. Systematic investigation of ginkgo biloba leaves for treating cardiocerebrovascular diseases in an animal model. ACS Chem Biol. 2017;12:1363-72.

10. Yu W, Li Z, Long F, Chen W, Geng Y, Xie Z, Yao M, Han B, Liu T. A systems pharmacology approach to determine active compounds and action mechanisms of Xipayi KuiJie'an enema for treatment of ulcerative colitis. Sci Rep. 2017;7.

11. Wang J, Li Y, Yang Y, Du J, Zhao M, Lin F, Zhang S, Wang B. Systems pharmacology dissection of multiscale mechanisms of action for herbal medicines in treating rheumatoid arthritis. Mol Pharm. 2017;14(9):3201-17.

12. Chun JM, Lee AY, Kim JS, Choi G, Kim SH. Protective effects of Peucedanum japonicum extract against osteoarthritis in an animal model using a 
combined systems approach for compound-target prediction. Nutrients. 2018;10(6).

13. Zeng L, Yang K, Ge J. Uncovering the pharmacological mechanism of Astragalus Salvia compound on pregnancy-induced hypertension syndrome by a network pharmacology approach. Sci Rep. 2017;7(1):16849.

14. Wheeler AP, Bernard GR. Acute lung injury and the acute respiratory distress syndrome: a clinical review. Lancet. 2007;369:1553-64.

15. Matthay MA, Goolaerts A, Howard JP, Lee JW. Mesenchymal stem cells for acute lung injury: preclinical evidence. Crit Care Med. 2010;38:S569-73.

16. Seeley JJ, Ghosh S. Molecular mechanisms of innate memory and tolerance to LPS. J Leukoc Biol. 2017;101:107-19.

17. Tian L, Li W, Wang T. Therapeutic effects of silibinin on LPS-induced acute lung injury by inhibiting NLRP3 and NF-kappa B signaling pathways. Microb Pathog. 2017;108:104-8.

18. Li C, Yang D, Cao X, Wang F, Jiang H, Guo H, Du L, Guo Q, Yin X. LFG-500, a newly synthesized flavonoid, attenuates lipopolysaccharide-induced acute lung injury and inflammation in mice. Biochem Pharmacol. 2016;113:57-69.

19. Butt $Y$, Kurdowska A, Allen TC. Acute lung injury: a clinical and molecular review. Arch Pathol Lab Med. 2016;140(4):345-50.

20. Chen H, Bai C, Wang X. The value of the lipopolysaccharide-induced acute lung injury model in respiratory medicine. Expert Rev Respir Med. 2010;4:773-83.

21. Matute-Bello G, Frevert CW, Martin TR. Animal models of acute lung injury Am J Physiol Lung Cell Mol Physiol. 2008:295(3):L379-99.

22. Kapoor K, Singla E, Sahu B, Naura AS. PARP inhibitor, olaparib ameliorates acute lung and kidney injury upon intratracheal administration of LPS in mice. Mol Cell Biochem. 2015;400(1-2):153-62

23. Wang X, Shen Y, Wang S, Li S, Zhang W, Liu X, Lai L, Pei J, Li H. PharmMapper 2017 update: a web server for potential drug target identification with a comprehensive target pharmacophore database. Nucleic Acids Res. 2017.

24. Wang JC, Chu PY, Chen CM, Lin JH. idTarget: a web server for identifying protein targets of small chemical molecules with robust scoring functions and a divide-and-conquer docking approach. Nucleic Acids Res. 2012;40:W393-9.

25. Kuleshov MV, Jones MR, Rouillard AD, Fernandez NF, Duan Q, Wang Z, Koplev S, Jenkins SL, Jagodnik KM, Lachmann A, McDermott MG, Monteiro $C D$, Gundersen GW, Ma'ayan A. Enrichr: a comprehensive gene set enrichment analysis web server 2016 update. Nucleic Acids Res. 2016; 44(W1):W90-7.

26. Bindea G, Mlecnik B, Hackl H, Charoentong P, Tosolini M, Kirilovsky A, Fridman WH, Pagès F, Trajanoski Z, Galon J. ClueGO: a Cytoscape plug-in to decipher functionally grouped gene ontology and pathway annotation networks. Bioinformatics. 2009;25(8):1091-3.

27. Szklarczyk D, Franceschini A, Wyder S, Forslund K, Heller D, Huerta-Cepas J, Simonovic M, Roth A, Santos A, Tsafou KP, Kuhn M, Bork P, Jensen LJ, von MC. STRING v10: protein-protein interaction networks, integrated over the tree of life. Nucleic Acids Res. 2015;43(Database issue):D447-52

28. Lv H, Zhu C, Liao Y, Gao Y, Lu G, Zhong W, Zheng Y, Chen W, Ci X. Tenuigenin ameliorates acute lung injury by inhibiting NF-KB and MAPK signalling pathways. Respir Physiol Neurobiol. 2015;216:43-51.

29. Wu H, Zhao G, Jiang K, Chen X, Zhu Z, Qiu C, Li C, Deng G. Plantamajoside ameliorates lipopolysaccharide-induced acute lung injury via suppressing NF-KB and MAPK activation. Int Immunopharmacol. 2016;35:315-22.

30. Yang X, Xu W, Huang K, Zhang B, Wang H, Zhang X, Gong L, Luo Y, He X. Precision toxicology shows that troxerutin alleviates ochratoxin $A$-induced renal lipotoxicity. FASEB J. 2019;33(2):2212-27.

31. Lu J, Wu D, Zheng Z, Zheng Y, Hu B, Zhang Z. Troxerutin protects against high cholesterol-induced cognitive deficits in mice. Brain. 2011;134:783-97.

32. Tang Y, Chen Y, Chu Z, Yan B, Xu L. Protective effect of cryptotanshinone on lipopolysaccharide-induced acute lung injury in mice. Eur J Pharmacol. 2014;723:494-500

33. Wei X, Li Y, Li M, Min C, Lu H, Li Q, Yuan J, Chen C, Li H, Zhang J. The protective effects of Sauropus spatulifolius on acute lung injury induced by lipopolysaccharide. J Sci Food Agric. 2018;98(12):4420-6.

34. Kibble M, Saarinen N, Tang J, Wennerberg K, Mäkelä S, Aittokallio T. Network pharmacology applications to map the unexplored target space and therapeutic potential of natural products. Nat Prod Rep. 2015;32(8):1249-66.

35. Hou J, Chen W, Lu H, Zhao H, Gao S, Liu W, Dong X, Guo Z. Exploring the therapeutic mechanism of Desmodium styracifolium on oxalate crystalinduced kidney injuries using comprehensive approaches based on proteomics and network pharmacology. Front Pharmacol. 2018;9(620).
36. Kim BY, Song KH, Lim CY, Cho SI. Therapeutic properties of Scutellaria baicalensis in $\mathrm{db} / \mathrm{db}$ mice evaluated using connectivity map and network pharmacology. Sci Rep. 2017;7(41711)

37. Li H, Sun X, Yu F, Xu L, Miu J, Xiao P. In silico investigation of the pharmacological mechanisms of beneficial effects of Ginkgo biloba L. on Alzheimer's disease. Nutrients. 2018;10(5).

38. Thomas NS, George K, AAA S: anticancer mechanism of troxerutin via targeting Nrf2 and NF-KB signalling pathways in hepatocarcinoma cell line. Toxicol in Vitro 2018, 54(317-329.

39. Thomas NS, George K, AAA S: Troxerutin subdues hepatic tumorigenesis via disrupting the MDM2-p53 interaction. Food Funct 2018, 9(10):5336-5349.

40. Shu L, Zhang W, Huang C, Huang G, Su G. Troxerutin protects against myocardial ischemia/reperfusion injury via Pi3k/Akt pathway in rats. Cell Physiol Biochem. 2017;44(5):1939-48.

41. Sampath S, Karundevi B. Effect of troxerutin on insulin signaling molecules in the gastrocnemius muscle of high fat and sucrose-induced type-2 diabetic adult male rat. Mol Cell Biochem. 2014;395:11-27.

42. A. Subastri, A. Suyavaran, P.B. E, N. S, R. Bharathidasan, C. Thirunavukkarasu, Troxerutin with copper generates oxidative stress in cancer cells: its possible chemotherapeutic mechanism against hepatocellular carcinoma, J Cell Physiol. (2017).

43. Baluchnejadmojarad T, Jamali-Raeufy N, Zabihnejad S, Rabiee N, Roghani M. Troxerutin exerts neuroprotection in 6-hydroxydopamine lesion rat model of Parkinson's disease: possible involvement of PI3K/ERß signaling. Eur J Pharmacol. 2017:801:72-8.

44. Salama SA, Arab HH, Maghrabi IA. Troxerutin down-regulates KIM-1, modulates p38 MAPK signaling, and enhances renal regenerative capacity in a rat model of gentamycin-induced acute kidney injury. Food Funct. 2018.

45. Yang X, Xu W, Huang K, Zhang B, Wang H, Zhang X, Gong L, Luo Y, He X. Precision toxicology shows that troxerutin alleviates ochratoxin A-induced renal lipotoxicity. FASEB J. 2018:fj201800742R.

46. Najafi M, Noroozi E, Javadi A, Badalzadeh R. Anti-arrhythmogenic and antiinflammatory effects of troxerutin in ischemia/reperfusion injury of diabetic myocardium. Biomed Pharmacother. 2018;102:385-91.

47. Fan SH, Zhang ZF, Zheng YL, Lu J, Wu DM, Shan Q, Hu B, Wang YY. Troxerutin protects the mouse kidney from $\mathrm{d}$-galactose-caused injury through antiinflammation and anti-oxidation. Int Immunopharmacol. 2009;9:91-6.

48. Goodman RB, Pugin J, Lee JS, Matthay MA. Cytokine-mediated inflammation in acute lung injury. Cytokine Growth Factor Rev. 2003;14:523-35.

49. Gattinoni L, Tonetti T, Cressoni M, Cadringher P, Herrmann P, Moerer O, Protti A, Gotti M, Chiurazzi C, Carlesso E, Chiumello D, Quintel M. Ventilatorrelated causes of lung injury: the mechanical power. Intensive Care Med. 2016;42(10):1567-75.

50. Mohammad HB, Bayrami G, Jalali S, Farkhondeh T. The effect of the extract of crocus Sativus on tracheal responsiveness to ovalbumin sensitized Guinea-pigs. Clin Biochem. 2011;44(13):S117-117S117.

51. Kim EK, Choi EJ. Pathological roles of MAPK signaling pathways in human diseases. Biochim Biophys Acta. 2010;1802:396-405.

52. Baker KE, Bonvini SJ, Donovan C, Foong RE, Han B, Jha A, Shaifta Y, Smit M, Johnson JR, Moir LM. Novel drug targets for asthma and COPD: lessons learned from in vitro and in vivo models. Pulm Pharmacol Ther. 2014;29:181-98.

53. Sethi GS, Dharwal V, Naura AS. Poly (ADP-ribose)Polymerase-1 in lung inflammatory disorders: a review. Front Immunol. 2017;8(1172).

54. Yu Y, Zheng G. Troxerutin protects against diabetic cardiomyopathy through NF-KB/AKT/IRS1 in a rat model of type 2 diabetes. Mol Med Rep. 2017;15:3473-8

\section{Ready to submit your research? Choose BMC and benefit from:}

- fast, convenient online submission

- thorough peer review by experienced researchers in your field

- rapid publication on acceptance

- support for research data, including large and complex data types

- gold Open Access which fosters wider collaboration and increased citations

- maximum visibility for your research: over $100 \mathrm{M}$ website views per year

At $\mathrm{BMC}$, research is always in progress.

Learn more biomedcentral.com/submissions 